\title{
Loss of Nix in Pdx1-deficient mice prevents apoptotic and necrotic $\beta$ cell death and diabetes
}

\author{
Kei Fujimoto, ${ }^{1}$ Eric L. Ford, ${ }^{1}$ Hung Tran, ${ }^{1}$ Burton M. Wice, ${ }^{1}$ Seth D. Crosby, ${ }^{2}$ \\ Gerald W. Dorn II, ${ }^{3}$ and Kenneth S. Polonsky' \\ 1Division of Endocrinology, Metabolism and Lipid Research, Department of Medicine, ${ }^{2}$ Genome Sequencing Center, Department of Genetics, \\ and ${ }^{3}$ Center for Pharmacogenomics, Department of Medicine, Washington University School of Medicine, St. Louis, Missouri, USA.
}

\begin{abstract}
Mutations in pancreatic duodenal homeobox (PDX1) are linked to human type 2 diabetes and maturity-onset diabetes of the young type 4 . Consistent with this, $P d x 1$-haploinsufficient mice develop diabetes. Both apoptosis and necrosis of $\beta$ cells are mechanistically implicated in diabetes in these mice, but a molecular link between Pdx1 and these 2 forms of cell death has not been defined. In this study, we introduced an shRNA into mouse insulinoma MIN6 cells to deplete $P d x 1$ and found that expression of proapoptotic genes, including NIP3-like protein X (Nix), was increased. Forced Nix expression in MIN6 and pancreatic islet $\beta$ cells induced programmed cell death by simultaneously activating apoptotic and mitochondrial permeability transition-dependent necrotic pathways. Preventing Nix upregulation during Pdx1 suppression abrogated apoptotic and necrotic $\beta$ cell death in vitro. In $P d x 1$-haploinsufficient mice, $N i x$ ablation normalized pancreatic islet architecture, $\beta$ cell mass, and insulin secretion and eliminated reactive hyperglycemia after glucose challenge. These results establish $\mathrm{Nix}$ as a critical mediator of $\beta$ cell apoptosis and programmed necrosis in Pdx1-deficient diabetes.
\end{abstract}

\section{Introduction}

Embryonic and postnatal development of the pancreas is orchestrated by the transcription factor pancreatic duodenal homeobox (PDX1; also known as insulin promoter factor 1, IPF1). Homozygosity of a frameshift PDX1 mutation that ablates the PDX1 DNA binding domain causes the rare clinical syndrome pancreatic agenesis (1), whereas heterozygous loss-of-function $P D X 1$ mutations are linked to common human type 2 diabetes and cause heritable maturity-onset diabetes of the young type 4 (2-4). These human diabetic syndromes are characterized by absolute or relative insulin insufficiency without pancreatic exocrine or other endocrine insufficiency, suggestive of a unique regulatory function of PDX1 in insulin-secreting pancreatic islet $\beta$ cells. Because they faithfully recapitulate the human clinical phenotypes, $P d x 1$-deficient mice have been widely used to characterize and define cellular mechanisms for $\beta$ cell loss in diabetes. Homozygous null $P d x 1$ mutations cause murine pancreatic agenesis, whereas $P d x 1$-haploinsufficient mice exhibit characteristically small pancreatic islets with greatly reduced numbers of $\beta$ cells, decreased insulin secretion, and glucose intolerance that results in diabetes (5-7).

Because Pdx1 directs pancreatic growth during development, the cellular basis for diabetes caused by $P d x 1$ deficiency was initially thought to be decreased pancreatic $\beta$ cell genesis $(6,8-10)$. Recent studies have refuted this notion, however, implicating programmed $\beta$ cell death rather than decreased proliferation as the causative event in diabetes caused by $P d x 1$ insufficiency. This issue has been made more complex as 3 mechanistically distinct pathways to programmed $\beta$ cell death have been observed in murine $P d x 1$ haploinsufficiency, apoptosis (11-14), autophagy (15), and programmed necrosis (16). Parallel pathways to $\beta$ cell death offer an explanation for intermediate phenotypes obtained when individual death pathways have been targeted in $P d x 1$-haploinsufficient mice $(15,16)$.

Conflict of interest: The authors have declared that no conflict of interest exists. Citation for this article: J Clin Invest. 2010;120(11):4031-4039. doi:10.1172/JCI44011.
The molecular basis by which relative insufficiency of $P d x 1$, a transcription factor that directs embryonic pancreatic growth and differentiation, induces multiple pathways of $\beta$ cell death is not known. Because pancreatic islet $\beta$ cells are dynamically regulated throughout life in response to metabolic stress (17), we considered that in addition to its developmental function, $\mathrm{Pdx} 1$ may play a critical homeostatic role in the adult pancreas, balancing $\beta$ cell proliferation and programmed elimination in accordance with metabolic need. According to this scenario, the $\beta$ cell growth factor Pdx1 should be regulated in opposition to as-yet-unidentified $\beta$ cell death factors. Here, we performed unbiased transcriptional profiling of cultured murine MIN6 $\beta$ cells after Pdx1 suppression by shRNA in search of reciprocal upregulation of programmed death genes and identified NIP3-like protein X (Nix; also known as BNip3L). Preventing Nix upregulation abrogated apoptotic and programmed necrotic $\beta$ cell death induced by $\mathrm{Pdx} 1$ deficiency in vitro, and ablation of the Nix gene was sufficient to fully prevent both the diabetic phenotype and the morphological abnormalities in $P d x 1$-haploinsufficient mouse islets.

\section{Results}

Transcriptional profiling of Pdx1-deficient cultured insulinoma cells. To identify gene dysregulation induced by $\mathrm{Pdx} 1$ insufficiency, we used microarrays to compare the transcriptional signatures of cultured MIN6 mouse insulinoma $\beta$ cells after Pdx1 suppression with our previously validated lentivirus-expressed $\operatorname{Pdx1} \operatorname{shRNA}(15,16)$. Nonbiased hierarchical clustering of the mRNA expression profile in MIN6 cells after Pdx1 suppression (Figure 1A) identified 56 upregulated and 14 downregulated mRNAs $(P<0.01$; FDR, 0.05). Grouping of differentially expressed transcripts by gene ontology category revealed that apoptosis/programmed cell death mRNAs were disproportionately represented among upregulated mRNAs (Figure 1B and Supplemental Table 1; supplemental material available online with this article; doi:10.1172/JCI44011DS1). Among 

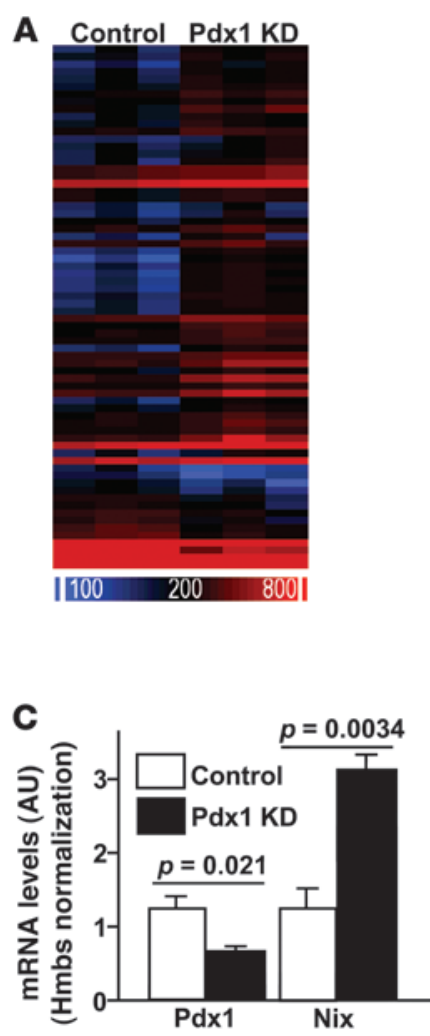

B

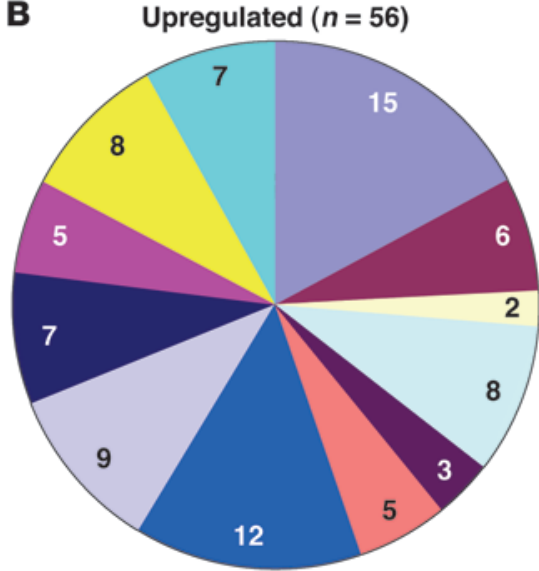

D

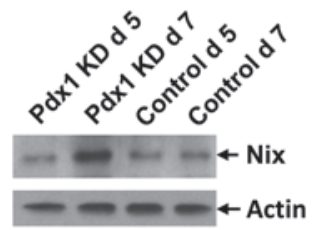

Downregulated $(n=14)$

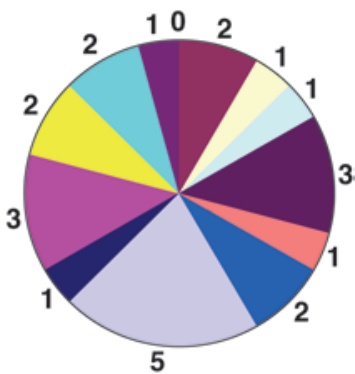

$\square$ apoptosis

- cell surface receptor linked signal

transduction

cytoskeleton

$\square$ endoplasmic reticulum

glucose metabolic process

lipid metabolic process

mitochondrial respiratory chain

$\square$ mitochondrion

nucleus

- intracellular signaling cascade

regulation of transcription

response to stress

translation

atransport

proteolysis

\section{Figure 1}

Pdx1 deficiency induces Nix expression in MIN6 cells. (A) Microarray analysis of mRNAs in control MIN6 cells and cells in which expression of Pdx1 was knocked down (KD) by exposing the cells to a lentivirus-containing shRNA to Pdx1 (3 independent experiments). Blue, low expression; red, high expression. (B) Gene ontology pie charts illustrating upregulated (left) and downregulated (right) genes. (C) Real-time PCR of $P d x 1$ and Nix mRNAs in Pdx1 knockdown MIN6 cells. Data represent mean \pm SEM; $n=6$ per group. (D) Immunoblot of Nix expression in Pdx1 knockdown and control MIN6 cells 5 or 7 days after lentiviral infection.

these upregulated transcripts were the proapoptotic Bcl2 family members Nix, Bim, Puma, and Bcl2l14 (Supplemental Table 1). Confirmatory quantitative real-time PCR demonstrated robust upregulation of Nix, Bim, Puma, and Bcl2l14 mRNAs (Figure 1C and Supplemental Figure 1). Immunoblot analysis showed a corresponding increase in Nix protein induced by $\mathrm{Pdx} 1$ suppression (Figure 1D). Nix upregulation preceded cell death (see below).

Nix induces programmed cell death in cultured insulinoma cells. Recent studies have implicated Nix as a transcriptionally regulated apical regulator of apoptosis, autophagy, and/or necrosis in erythropoietic and cardiac cells (18-24). To our knowledge, its presence in pancreatic $\beta$ cells has not previously been described. For this reason, we determined the consequences of increased Nix expression on this cell type: Nix was expressed in MIN6 cells in which Pdx1 was not suppressed (Figure 1D). Using an adenoviral (Ad) vector (22) at MOI of 10, we detected Flag-tagged Nix immunoreactivity in $95 \% \pm 5 \%$ of MIN6 cells (Figure 2A). Consistent with its reported ability to stimulate the mitochondrial apoptosis pathway in a Bax/Bak-dependent manner (23), Nix localized largely to a mitochondrial-enriched subcellular fraction labeled by cytochrome C oxidase IV antibody (COX IV) and with mitochondria, stimulated the release of cytochrome $\mathrm{c}$ into the cytoplasm, and induced caspase- 3 activation without induction of autophagy, measured as accumulation of LC3-II (Figure 2, B-E).
Although apoptosis is the mechanism of programmed $\beta$ cell death most often reported with $P d x 1$ deficiency (11-14), we recently discovered that programmed necrosis mediated by opening of the mitochondrial permeability transition pore (MPTP) also plays a role in the diabetes of murine $P d x 1$ haploinsufficiency (16). Therefore, we determined if Nix induced the mitochondrial permeability transition in cultured murine $\beta$ cells. MIN6 cells were loaded with rhodamine 123 , a mitochondrial dye whose fluorescence depends upon mitochondrial membrane potential $\left(\Delta \psi_{\mathrm{m}}\right)$. MPTP opening dissipates $\Delta \psi_{\mathrm{m}}$, and loss of rhodamine 123 fluorescence can therefore indicate MPTP opening (16). Figure $2 \mathrm{D}$ illustrates loss of rhodamine 123 fluorescence in Nix-overexpressing MIN6 cells. Quantitative analysis by fluorescence cell sorting revealed an approximately 50\% loss of rhodamine 123 fluorescence in 3 independent studies (Figure 2F). Collectively, the above studies show that Nix activates apoptosis and necrosis pathways in cultured $\beta$ cells.

We interrogated the roles of apoptosis and necrosis in Nixmediated $\beta$ cell death using propidium iodide (PI) staining as a marker for both apoptotic and necrotic cell death, and comparing the effects of pharmacological inhibitors of apoptosis (the caspase- 3 inhibitor DEVD-CHO) and MPTP-dependent necrosis (the MPTP blocker cyclosporin A). Ad-Nix increased PI staining, and this was almost completely prevented by both DEVD-CHO and cyclosporin A (Figure $2 \mathrm{G}$ ). 
A
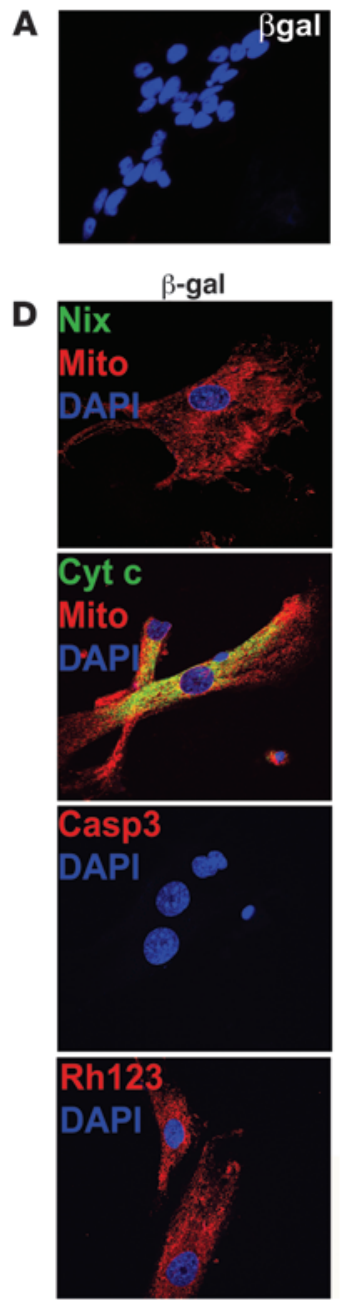

G
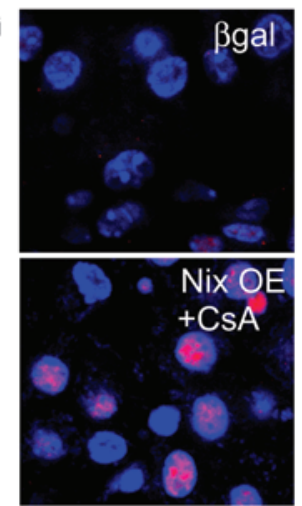

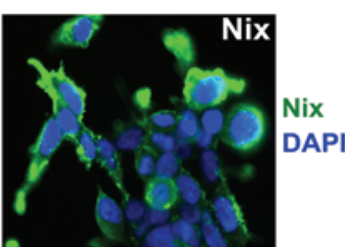

Nix OE
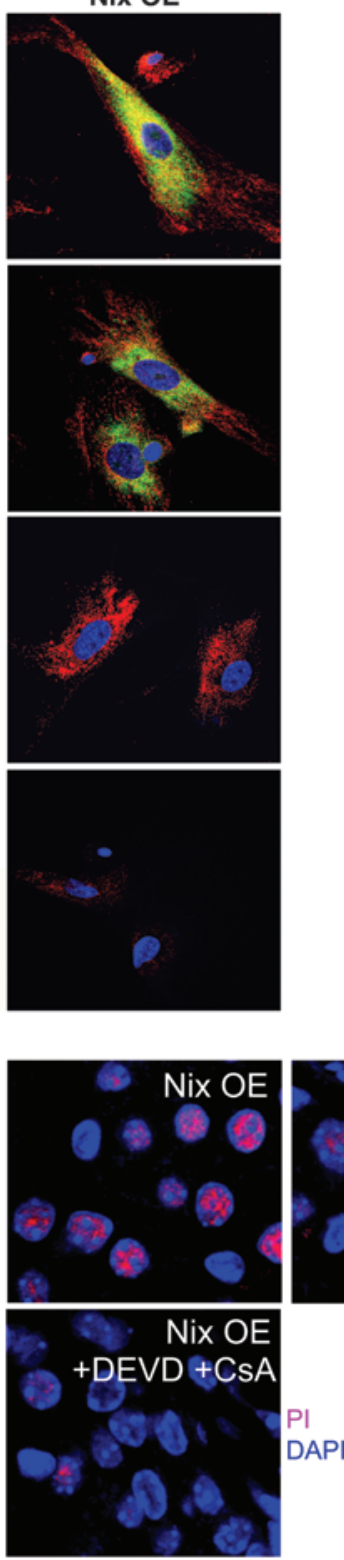

B

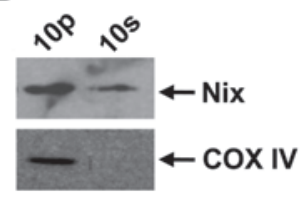

E

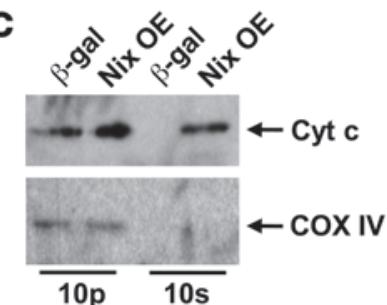

$\mathbf{F}$
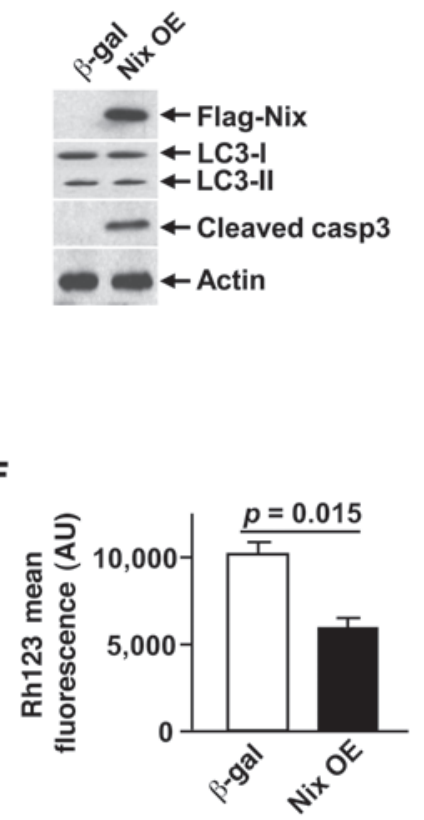

\section{Figure 2}

Nix overexpression induces programmed MIN6 cell death. (A) MIN6 cells with overexpression (OE) of $\beta$-gal or Flag-tagged Nix were stained with anti-Flag antibody (green) and DAPI (blue). Original magnification, $\times 400$. (B) MIN6 cells were fractionated into 10,000 $g$ pellet (10p) or $10,000 \mathrm{~g}$ supernatant (10s) and immunoblotted with antibody against Nix or COX IV, a marker of the mitochondrial fraction. (C) MIN6 cells with overexpression of $\beta$-gal or Nix were fractionated as in B, followed by immunoblotting with anti-cytochrome c (Cyt c) or COX IV antibodies. (D) Confocal imaging of $\beta$-gal- or Nix-overexpressing MIN6 cells. First row, Flag-tagged Nix (green) and mitochondrial (red) colocalization; second row, cytochrome c (green) and mitochondrial (red) staining; third row, caspase-3 activity (red); fourth row, rhodamine 123 (Rh123) staining (red). DAPI staining (blue) is also shown. Original magnification, $\times 1,000$. (E) Immunoblot of Flag-tagged Nix, LC-3, and cleaved caspase-3 in $\beta$-gal- and Nix-overexpressing MIN6 cells. (F) Fluorescence cell sorting analysis of rhodamine 123 in $\beta$-gal- and Nix-overexpressing MIN6 cells. Mean fluorescence of data from 3 independent experiments is shown. (G) PI (red) and DAPI (blue) staining in $\beta$-gal- and Nix-overexpressing MIN6 cells as a function of $10 \mu \mathrm{M}$ DEVD-CHO and/or $1 \mathrm{nM}$ cyclosporin A (CsA) treatment. Quantitation of 3 independent experiments for PI staining data is shown at right. Original magnification, $\times 600$. Data represent mean \pm SEM. 
A

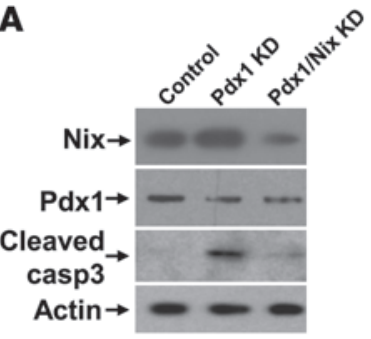

C

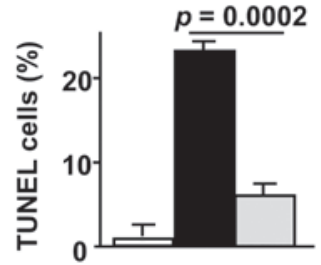

D

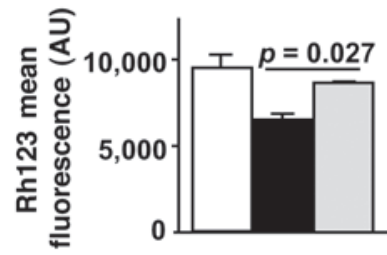

E

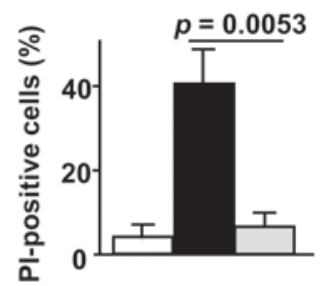

B
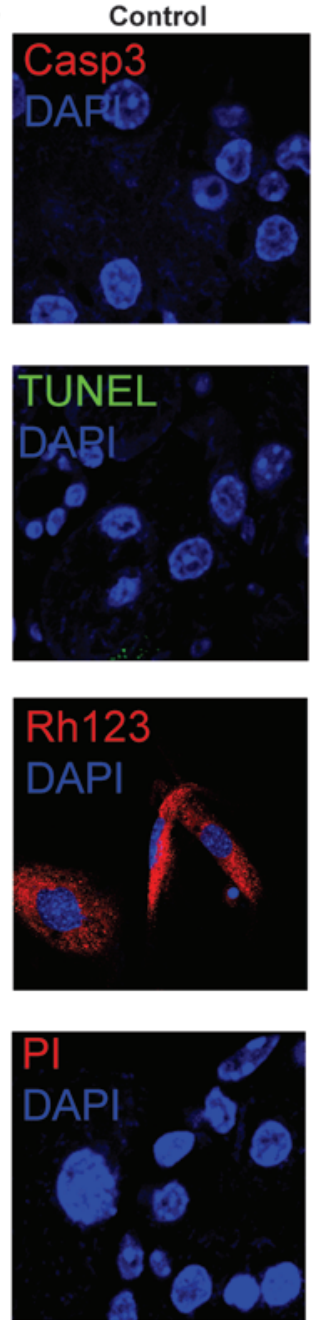

Pdx1 KD
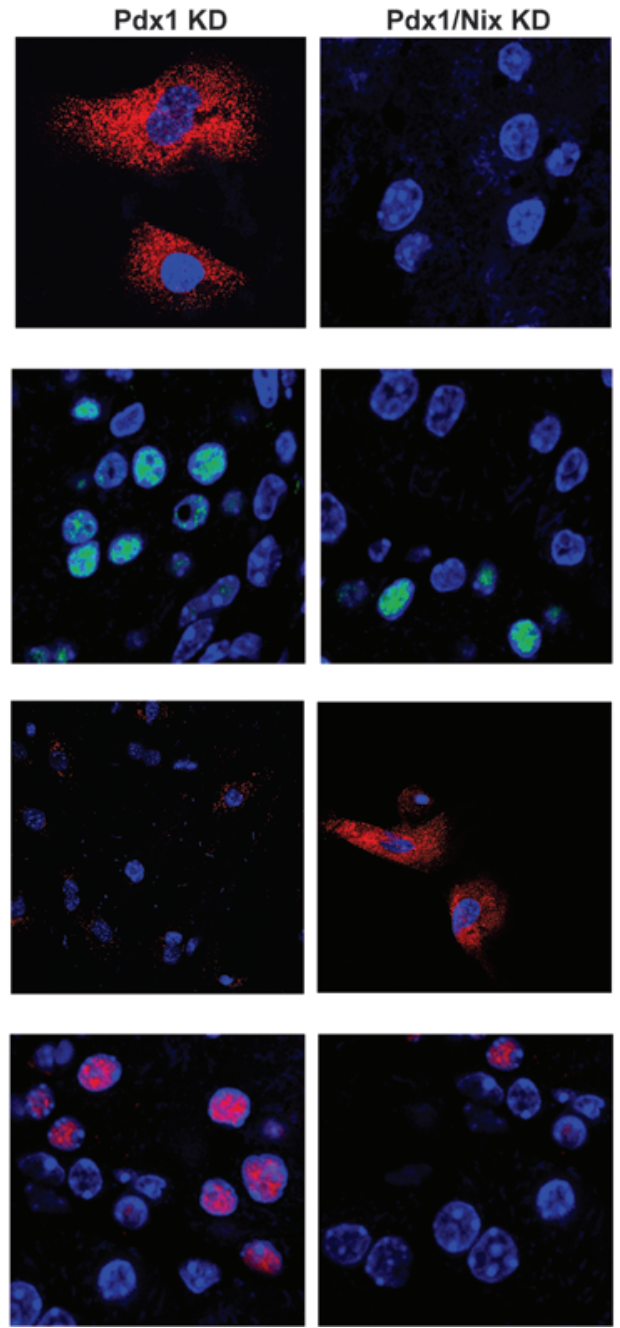

\section{Figure 3}

Nix upregulation is necessary for MIN6 cell death induced by Pdx1 suppression. (A) Immunoblot of Nix, Pdx1, and cleaved caspase-3 in control, Pdx1 knockdown, and Pdx1/Nix knockdown MIN6 cells. (B) Active caspase-3 staining in control, Pdx1 knockdown, and Pdx1/Nix knockdown MIN6 cells. (C) TUNEL labeling (green) with DAPI (blue) in control, Pdx1 knockdown, and Pdx1/Nix knockdown MIN6 cells. Quantitation of 3 independent experiments is shown at left. (D) Rhodamine 123 staining (red) with DAPI (blue) in control, Pdx1 knockdown, and Pdx1/Nix knockdown MIN6 cells. Mean fluorescence of 3 independent experiments is shown at left. (E) PI (red) and DAPI (blue) staining in control, Pdx1 knockdown, and Pdx1/Nix knockdown MIN6 cells. Quantitation of 3 independent experiments is shown at left. Original magnification, $\times 1,000$. Data represent mean \pm SEM.

Nix upregulation is necessary for $\beta$ cell death induced by $P d x 1$ suppression. The above results describe an association between $\mathrm{Pdx} 1$ downregulation and Nix upregulation and demonstrate that Nix was sufficient to cause apoptotic and necrotic death of cultured $\beta$ cells. To determine whether Nix upregulation after $\mathrm{Pdx} 1$ suppression is the essential event inducing MIN6 cell death after Pdx1 knockdown, we used shRNA to suppress Nix in cells infected with the Pdx1 shRNA lentivirus. Nix suppression in the $\mathrm{Pdx} 1$-insufficient state prevented caspase- 3 activation, TUNEL positivity, loss of $\Delta \psi_{\mathrm{m}}$, and cell death as assessed by PI staining (Figure 3, A-E). Collectively, these and the above-described findings showed that Nix is both necessary and sufficient for programmed MIN6 apoptotic and necrotic death induced by acute $\mathrm{Pdx} 1$ suppression.

Nix is upregulated in Pdx1-haploinsufficient pancreatic islets and induces programmed islet cell death. To determine whether Nix induction plays a role in $\beta$ cell loss seen with murine $P d x 1$ haploinsuf- ficiency, we assayed Nix mRNA levels in pancreatic islets isolated from $P d x 1^{+/-}$mice fed a high-fat diet. Despite having proportionately fewer $\beta$ cells than normal islets (see below), Nix mRNA levels indexed to the housekeeping gene $\mathrm{Hmbs}$ increased more than 2 -fold in $P d x 1^{+/-}$compared with control islets (Figure 4A). This recapitulates the observation from MIN6 cells that Pdx1 and Nix are antithetically regulated and shows that this regulatory pairing is maintained in the context of chronic germline $P d x 1$ insufficiency. Consistent with a role for Nix upregulation in $\beta$ cell death, apoptotic and necrotic death were induced in normal $\left(P d x 1^{+/+}\right)$isolated pancreatic islets infected with Ad-Nix (MOI of 10; 85\% $\pm 4 \%$ expression; Figure 4B), as assessed by caspase- 3 activation and dissipation of mitochondrial $\Delta \psi_{\mathrm{m}}$ (Figure $4, \mathrm{C}$ and D).

Nix ablation preserves $\beta$ cell mass and islet architecture in Pdx1-haploinsufficient mice. Because Nix was upregulated by Pdx1 deficiency in isolated pancreatic islets and cultured MIN6 cells, and increased 
A

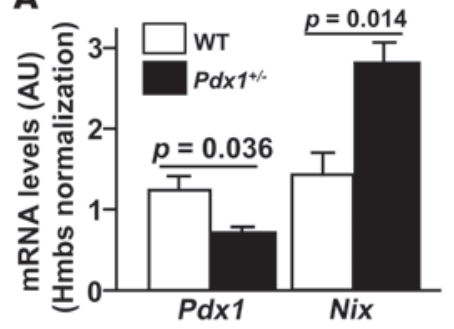

C

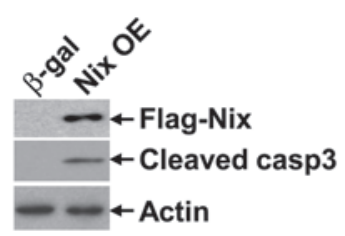

B
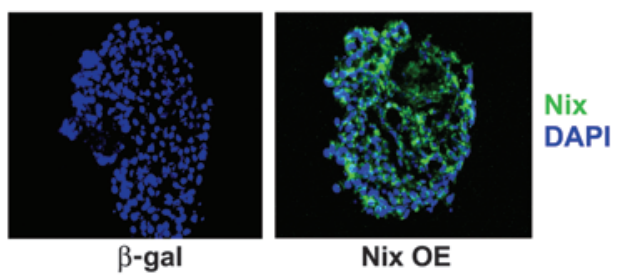

D

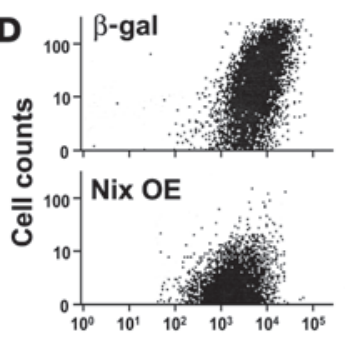

Fluorescence intensity (AU)

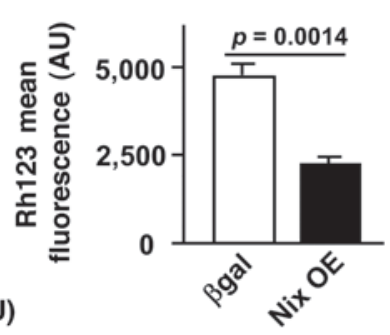

Figure 4

Nix overexpression induces programmed islet cell death. (A) Real-time PCR of mRNA for Pdx1 and Nix using islets from WT and $P d x 1^{+/-}$mice after 1 week on a highfat diet ( $n=5-6$ each). (B) Normal mouse islets expressing $\beta$-gal or Flag-tagged Nix were stained with anti-Flag antibody (green) and DAPI (blue). Original magnification, $\times 400$. (C) Immunoblot of Flag-tagged Nix and cleaved caspase-3 in mouse islets overexpressing $\beta$-gal or Nix. (D) Cell sorting analysis to detect rhodamine 123 fluorescence in $\beta$-gal- or Nix-overexpressing islet cells. Mean fluorescence of 3 independent experiments is shown at right. Data represent mean \pm SEM.
Nix expression caused programmed $\beta$ cell death in these systems, we determined whether preventing $\beta$ cell Nix upregulation in $P d x 1^{+/-}$mice also prevents $\beta$ cell loss in the in vivo condition. To accomplish this, we crossed $P d x 1^{+/-}$mice onto the Nix-null $\left(\mathrm{Nix}^{-/-}\right)$ background (21) and performed comparative histological studies

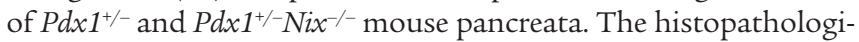
cal hallmarks of diabetes in the $P d x 1^{+/-}$mouse were small pancreatic islets containing reduced numbers of insulin-containing $\beta$ cells and abnormal islet architecture (Figure 5 and refs. 10, 15, 16). Whereas WT and $\mathrm{Nix}^{-/-}$pancreatic islets had a central core of $\beta$ cells ringed by a mantle of $\alpha$ cells, the small $P d x 1^{+/-}$islets had $\alpha$ cells distributed throughout the core, resulting in a characteristic decrease in absolute $\beta$ cell area and in the $\beta$ cell $/ \alpha$ cell ratio (Figure 5A) with no change in islet number per pancreas (data not shown). $P d x 1^{+/-}$ Nix ${ }^{-/-}$mice had normal $\beta$ cell area and $\beta$ cell/ $\alpha$ cell ratio, with normal islet architecture of a central core of $\beta$ cells surrounded by a mantle of $\alpha$ cells (Figure 5A). The area of $\beta$ cells per pancreas area on normal chow and high-fat diets was not different in $\mathrm{Nix}^{-/-}$mice compared with WT mice (Supplemental Figure 2A). Programmed $\beta$ cell death, as assessed by the number of TUNEL-positive $\beta$ cells, was characteristically increased in islets from $P d x 1^{+/-}$mice and strikingly reduced in those of $\mathrm{Pdx1^{+/- } \mathrm { Nix } x ^ { - / } \text { mice (Figure 5B). Nix }}$-/- islets showed TUNEL positivity comparable to that of WT mice (Figure $5 \mathrm{~B})$, which supports our conclusion that prevention of $\beta$ cell death in $\mathrm{Pdx} \mathrm{1}^{+/-} \mathrm{Nix} \mathrm{x}^{-/-}$mice is caused by Nix deficiency and not epiphenomena. These findings were consistent in adult mice fed a high-fat diet as well as in neonatal mice 1 day after birth (Figure 5, C and D), which indicates that increased Nix expression adversely influences islets during $P d x 1^{+/-}$mouse embryonic development, and not just during reactive islet growth in the adult.

Nix gene ablation prevents diabetes in Pdx1-haploinsufficient mice. To determine whether $\beta$ cell salvage by Nix ablation prevents the diabetic phenotype of $P d x 1^{+/-}$mice, studies of glucose and insulin tolerance were performed on $P d x 1^{+/-}$and $P d x 1^{+/-} \mathrm{Nix}^{-/-}$mice maintained on a high-fat diet to provoke glucose intolerance $(15,16) . P d x 1^{+/-}$ mice exhibited characteristic glucose intolerance and decreased basal and reactive insulin levels (Figure 6, A-C). In comparison,

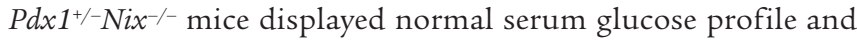

insulin response. On the other hand, $\mathrm{Nix}^{-/-}$mice showed comparable insulin secretion and glucose tolerance to that of WT mice fed normal chow and high-fat diets (Supplemental Figure 2, B and C). Since the $\mathrm{Nix}^{-/-}$mouse is a germline knockout (21), it is possible that increased insulin sensitivity contributed to normalization of the response to glucose challenge. However, insulin tolerance tests were similar in both groups (Figure 6D). Thus, we conclude that Nix ablation prevents the diabetic phenotype in $P d x 1$-haploinsufficient mice by augmenting insulin secretion.

\section{Discussion}

These studies define a single molecular mechanism for increased pancreatic $\beta$ cell apoptosis and programmed necrosis induced by $\mathrm{Pdx} 1$ insufficiency. Using the unbiased approach of transcriptional profiling in Pdx1-suppressed mouse insulinoma cells, we identified a molecular program for $\beta$ cell suicide that included Nix. We show that Nix activated $\beta$ cell signaling pathways, leading to caspasedependent apoptosis and MPTP-dependent necrosis, and that preventing Nix upregulation induced by $\mathrm{Pdx} 1$ deficiency was sufficient to prevent apoptotic and necrotic programmed $\beta$ cell death in vitro and in vivo. In the $P d x 1$-haploinsufficient mouse model of diabetes, Nix gene ablation corrected the characteristic small size and loss of $\beta$ cells from pancreatic islets, consequently preserving basal and reactive insulin secretion and normalizing glucose tolerance.

The current findings indicate that Nix and $\mathrm{Pdx} 1$ are opposing forces within a homeostatic mechanism that maintains $\beta$ cell mass through reciprocal modulation of proliferation and programmed elimination. Pdx1 stimulates pancreatic $\beta$ cell proliferation. Indeed, $\operatorname{Pdx} 1$ expression is essential for embryonic development of the pancreas, and homozygous loss-of-function mutations result in pancreatic agenesis in humans and mice $(1,5)$. In our prior studies of adult murine pancreatic islets, heterozygosity of $P d x 1$ dramatically depresses the rate of normal $\beta$ cell proliferation, measured as Ki67 labeling $(15,16)$. The second hit that limits $\beta$ cell mass in $P d x 1$ deficiency is a striking increase in programmed $\beta$ cell death, commonly designated as apoptosis because of caspase activation and increased TUNEL labeling $(15,16)$. However, caspase activity and TUNEL positivity simply reflect cytochrome c release from 
A
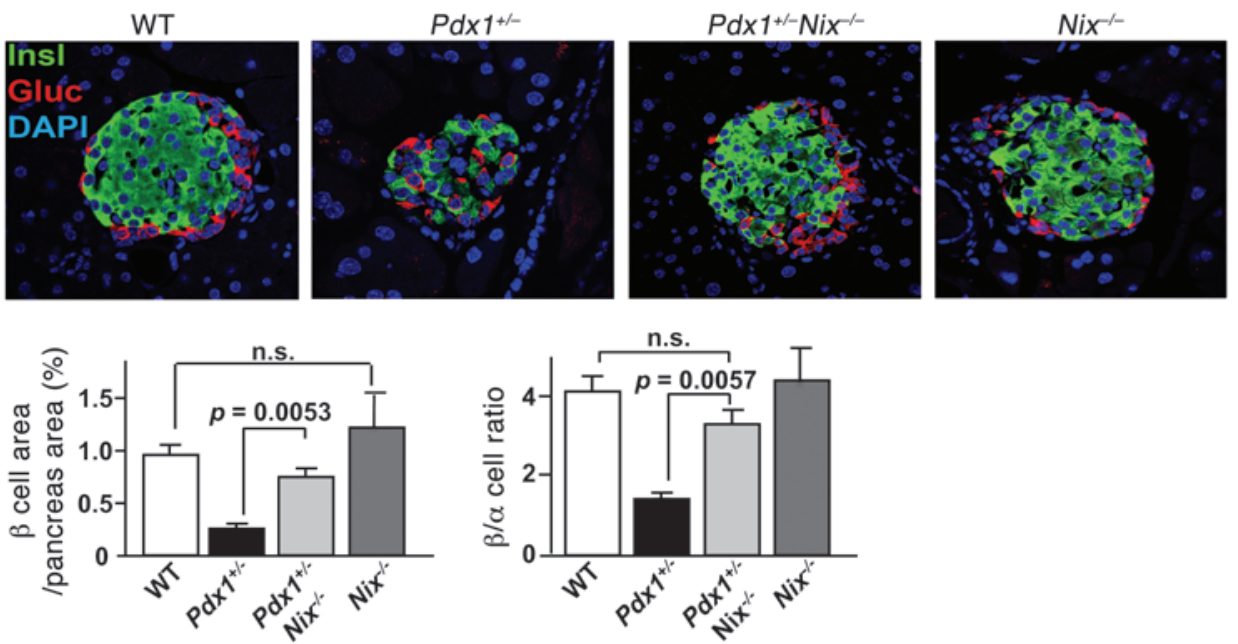

B
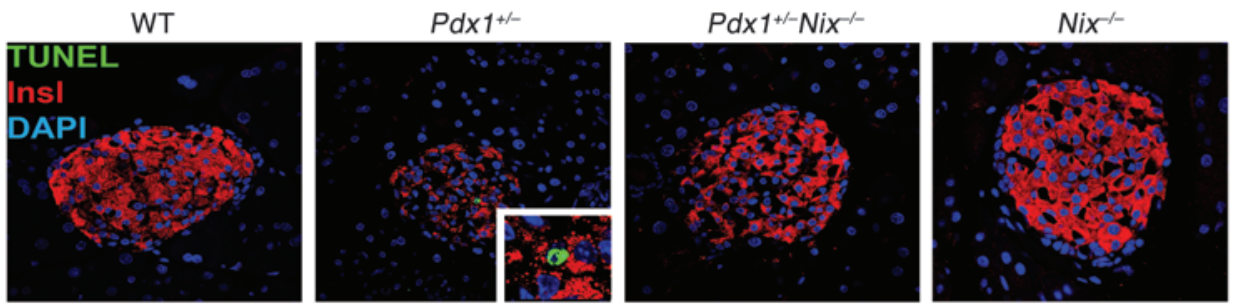

C
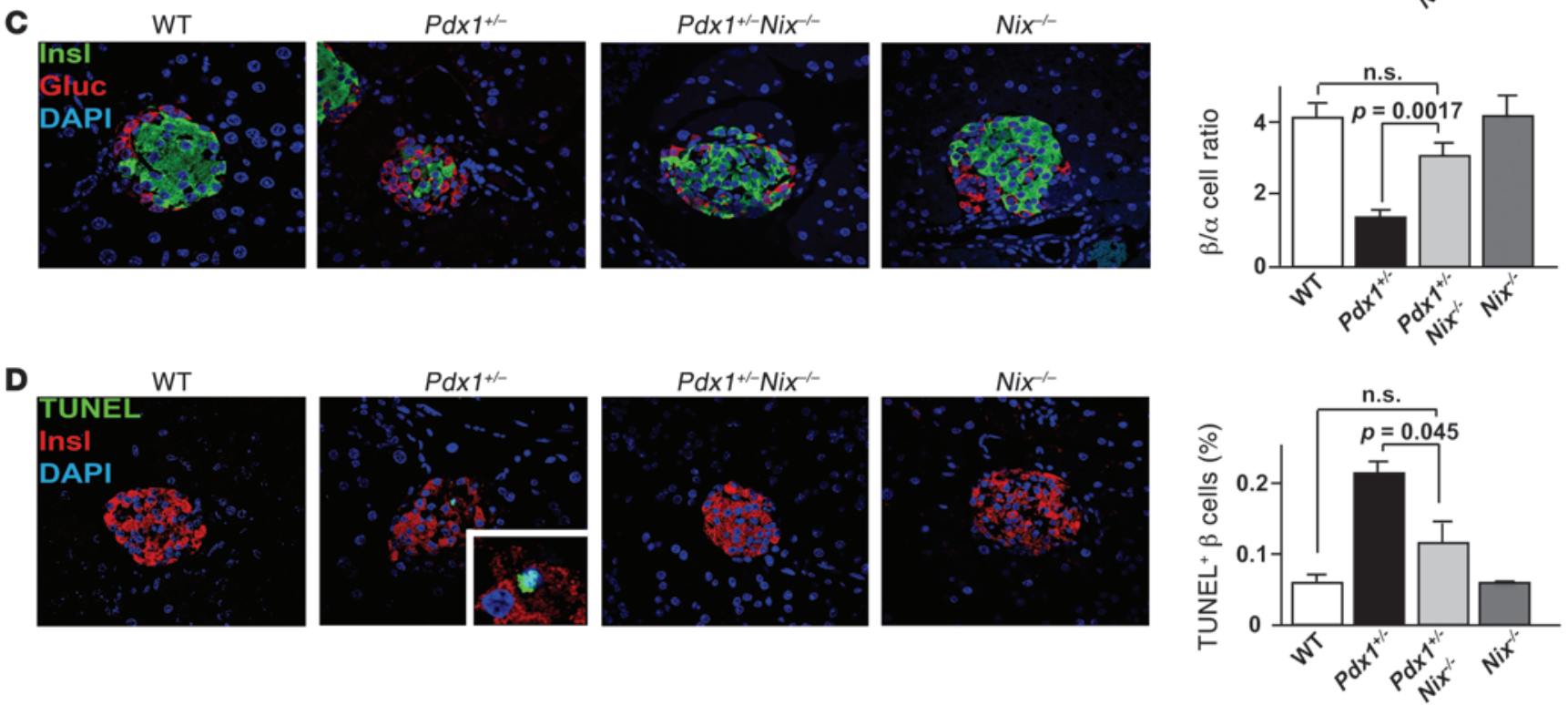

\section{Figure 5}

Preservation of $\beta$ cell mass and islet architecture in $\mathrm{Pdx} 1^{+/-} \mathrm{Nix}{ }^{-/}$mice. (A) Islet morphology in adult WT, $\mathrm{Pdx} 1^{+/-}, \mathrm{Pdx} 1^{+-} \mathrm{Nix}{ }^{-/}$, and $\mathrm{Nix}{ }^{-/-}$mice (9-11 weeks of age) after 6-8 weeks on a high-fat diet; $\alpha$ cells are stained red (anti-glucagon) and $\beta$ cells are stained green (anti-insulin). Quantitation of group data for $\beta$ cell area per pancreas area and $\beta$ cell/ $\alpha$ cell ratio are shown $(n=4-6$ per group). (B) TUNEL labeling of adult pancreatic $\beta$ cells; quantitative TUNEL data are shown at right $(n=5$, each group). (C) Pancreatic islets from 1-day-old mice comparing $\alpha$ cells (red; anti-glucagon) and $\beta$ cells (green; anti-insulin); quantitative group data are shown at right ( $n=5$ per group). (D) TUNEL labeling of neonatal $\beta$ cells; quantitative data are shown at right ( $n=5$ per group). Original magnification, $\times 600$ (including insets). Data represent mean \pm SEM. 

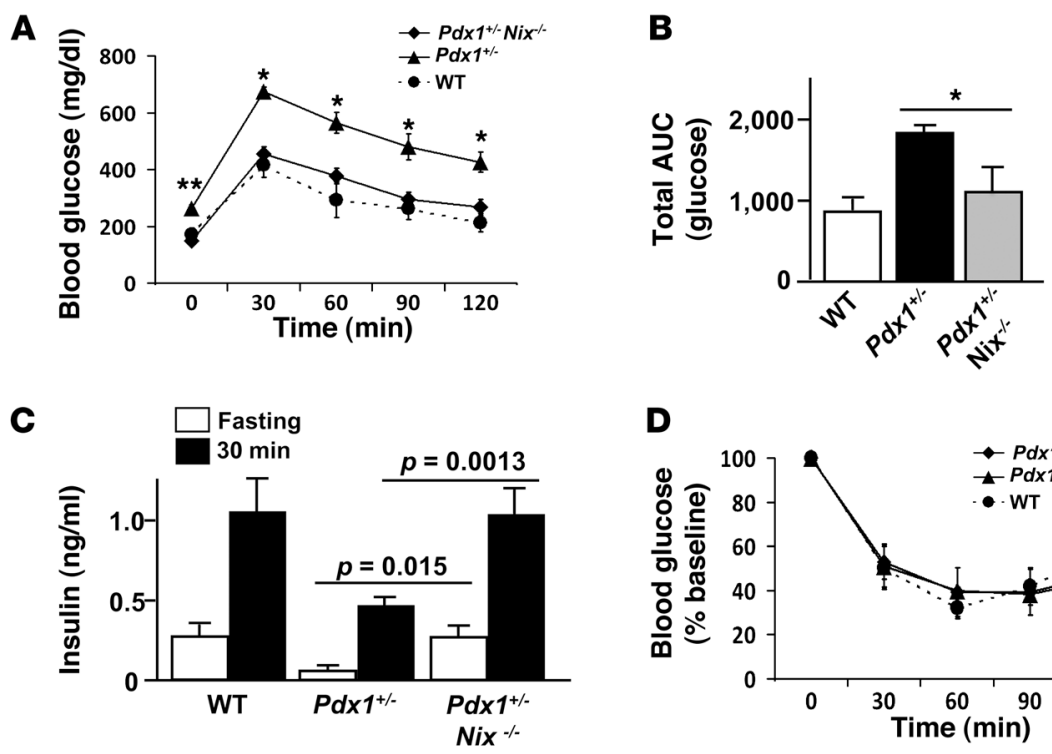

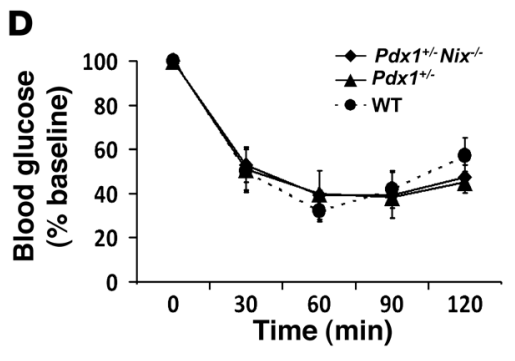

\section{Figure 6}

Genetic ablation of Nix prevents diabetes

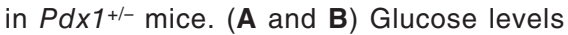
(A) and respective areas under the glucose curves (B) following intraperitoneal injection of $1 \mathrm{~g} / \mathrm{kg}$ dextrose. $n=7\left(P d \times 1^{+/-}\right) ; 10$ $\left(P d x 1^{+-} \mathrm{Nix}^{-/-}\right) ; 4$ (WT). ${ }^{*} P<0.01,{ }^{\star \star} P<0.001$, $P d x 1^{+/-}$versus $P d x 1^{+-} \mathrm{Nix}^{-1-}$. (C) Insulin levels measured fasting and 30 minutes after intraperitoneal dextrose. $n=7\left(P d x 1^{+/}\right) ; 10$ $\left(P d x 1^{\left.+/-N i x^{-l-}\right) ;} 4\right.$ (WT). (D) Glucose levels following administration of $0.75 \mathrm{U} / \mathrm{kg}$ insulin. $n=4$ per group. All group data are mean \pm SEM of $9-11$ weeks of age. mitochondria, which can result from outer membrane permeabilization (apoptosis) or from mitochondrial disruption as a consequence of swelling after MPTP opening (necrosis). Thus, TUNEL positivity should not be equated with apoptotic cell death. Indeed, we recently demonstrated that MPTP-dependent programmed $\beta$ cell necrosis is an important contributory mechanism in the diabetes of murine $P d x 1$ haploinsufficiency (16). Here, we identified Nix as a critical death-promoting counterregulatory factor that opposes $\beta$ cell proliferation induced by $\mathrm{Pdx} 1$. Indeed, suppression or genetic ablation of Nix was sufficient to prevent $\beta$ cell apoptosis and programmed necrosis induced by $\mathrm{Pdx} 1$ deficiency.

Nix is 1 of only 4 members of the BH3-only-like subfamily of $\mathrm{Bcl} 2$-like mitochondrial death proteins. The functional characteristic that distinguishes pseudo-BH3-only factors (Nix, BNip1, $\mathrm{BNip} 2$, and $\mathrm{BNip} 3$ ) from the larger group of true $\mathrm{BH} 3$-only factors (Bim, Puma, Bcl2114, and others) is induction of a unique form of cell death with features of both apoptosis and necrosis (22-25). The mechanism for dual pathway activation by Nix has recently been elucidated in detail (23). Nix localizes to mitochondrial outer membranes stimulates Bax/Bak-dependent outer membrane permeabilization, releasing cytochrome $\mathrm{c}$ into the cytosol, and initiating the intrinsic caspase cascade that leads to apoptosis $(19,21-25)$. Nix can also localize to ER, stimulating the Bax/Bak-independent activation of mitochondrial permeability pores via ER-mitochondrial calcium transfer. Opening of MPTP and consequent loss of $\Delta \psi_{\mathrm{m}}$ reverses oxidative phosphorylation, which depletes ATP, results in generation of ROS, and ultimately produces cellular necrosis from metabolic shutdown (22-25). The rare ability of Nix to activate independent apoptotic and necrotic pathways provides a mechanistic explanation for the complete prevention of the $P d x 1^{+/-}$diabetic phenotype through Nix gene ablation, whereas inhibition of programmed necrosis alone (via genetic ablation of the essential MPTP protein cyclophilin D) provided only partial prevention (16).

A third mechanism of programmed $\beta$ cell death, autophagy, has recently been implicated in $P d x 1$ deficiency (15). Autophagy induced by intense metabolic need (i.e., starvation) is a compensatory process enabling processing of cellular proteins for acute energy production $(15,25)$. An autophagic marker, subcellular aggregation of LC3 pro- tein (a constituent of autophagosomal membranes), is increased in Pdx1-deficient $\beta$ cells in vitro and in vivo, and inhibition of autophagy by Beclin 1 suppression or hemizygous gene ablation enhances Pdx1-deficient $\beta$ cell viability (15). However, the preponderance of data suggests that autophagy is a contributory factor, and not causative, in $\beta$ cell death caused by $\mathrm{Pdx} 1$ insufficiency. Inhibition of autophagy delays, but does not prevent, $\beta$ cell death (15), and the benefits of Nix ablation on survival of Pdx1-deficient $\beta$ cells were not associated with any decrease in autophagic markers in vitro or in vivo (Figure 2E and Supplemental Figure 3).

The current studies demonstrated counterregulation of $\beta$ cell mass by Pdx 1 and Nix in adult pancreata after the metabolic stress of a high-fat diet as well as in neonatal pancreata not been subjected to any such metabolic stress. Thus, it is likely that Pdx1 and Nix modulate reactive islet $\beta$ cell hyperplasia as well as the latter stages of embryonic pancreas development (26). Further support for an important developmental role for Nix is normalization of islet cellular architecture. Normal pancreatic islets consist of a central core of insulin-containing $\beta$ cells surrounded by a peripheral mantle of glucagon-containing $\alpha$ cells as well as other non-insulin-secreting cells. This highly ordered islet architecture is characteristically disturbed in $P d x 1$-deficient mice, in which loss of $\beta$ cells is associated with more random distribution of $\alpha$ cells throughout the islet core (ref. 16 and the present study). Moderation of $\beta$ cell death and increased islet size is not necessarily linked with preservation of normal peripheral $\alpha$ cell positioning, as recently shown in $P d x 1^{+/-}$Ppif ${ }^{\prime-}$ mice (16). However, in the current study, Nix ablation normalized pancreatic islet architecture as well as size and $\beta$ cell content. Thus, regulated expression of Nix is integral to islet development and maintenance.

The regulatory role we identified herein for $\mathrm{Pdx} 1 / \mathrm{Nix}$ pairing in pancreatic islet $\beta$ cell homeostasis is similar to that previously described for erythropoietin and Nix in erythrocyte (red blood cell) formation from erythroid precursor cells $(21,27)$. The process of erythroblast proliferation and progression down the differentiation pathway to erythroblasts is stimulated by erythropoietin in response to metabolic need (hypoxia or decreased red blood cell mass). Nix and antiapoptotic Bcl-xL are transcriptionally upregulated in response to erythropoietin during erythroid differentiation from 
erythroblasts. The balance between $\mathrm{Bcl}-\mathrm{xL}$ and Nix expression regulates erythroblast survival, and therefore the quantity of cells that progress to fully developed erythrocytes released into the peripheral circulation. In this scenario, erythropoietin is acting on the erythroid lineage in the same manner as $\mathrm{Pdx} 1$ acts for $\beta$ cells, as a molecule that transmits a specific form of physiological stress into a message to induce the proper target cell proliferation. Nix plays the same functional role in both pathways: balancing target cell proliferation with programmed elimination. Maintaining homeostasis in cells that are required to undergo context-specific proliferation is a general paradigm that can explain the very presence of programmed death genes in these tissues. Accordingly, the approach of interrupting programmed cell death offers therapeutic potential in any condition where normal homeostasis is unbalanced.

The mechanism by which $\mathrm{Pdx} 1$ deficiency regulates Nix expression likely involves 1 of the 2 pathways previously described for Nix transcriptional regulation, PKC and $\operatorname{HIF} 1(28,29)$. Pdx1 targets PKC $\alpha$ and PKCE (28), and PKC $\alpha$ was previously shown to direct Nix expression in the heart (30). Pdx1 also regulates HIF1 (29), and HIF1 increases Nix expression in several tissues (31-33). We believe that $\mathrm{Pdx} 1$ regulation of $\mathrm{Nix}$ is complex and involves multiple factors. These issues are currently being addressed in additional studies.

In conclusion, we used unbiased discovery of Pdx1-regulated transcripts, gene manipulation in cultured insulinoma cells and mouse islets, and genetic complementation studies in intact mice to identify Nix as a critical molecular effector of diabetes in $P d x 1$ insufficiency. Nix induces 2 mitochondrial cell death pathways, apoptosis and programmed necrosis, in Pdx1-deficient $\beta$ cells. Not only do these results explain diverse mechanisms of $\beta$ cell loss as a result of $P d x 1$ deficiency, but they suggest a single molecular target for preventing diabetes caused by abnormal $\beta$ cell death.

\section{Methods}

Microarrays. mRNA from 3 independent wells of MIN6 cells infected with Pdx1 shRNA was compared with control infected cells. MIN6 cell cDNA was prepared followed by hybridization to microarrays (Illumina MouseRef6 BeadChips, which cover approximately 48,000 transcripts derived from the MEEBO set, and RIKEN FANTOM 2).

Hierarchical clustering. Normalized expression data for mRNAs were subjected to unsupervised hierarchical clustering to provide a visual assessment of the mRNA profiles. Agglomerative hierarchical clustering was performed using Partek software (Partek Inc.). Hierarchical clustering was used to group similar objects into clusters; the algorithm represents the similarity between clusters. The methodology for this technique was described previously (34). In our experiments, Pearson distance was used to measure dissimilarity (the distance between 2 rows or columns), and average linkage (the average distance between all pairs of objects in 2 different clusters) was used as the measure of distance between 2 clusters.

Gene ontology. The official AmiGO tool (http://amigo.geneontology. org/cgi-bin/amigo/go.cgi) was used to search the gene ontology database (http://www.geneontology.org/), which consists of a controlled vocabulary of terms covering biological concepts and a large number of genes.

PI/DAPI cell death assay. For the last hour of incubation, $10 \mu \mathrm{g} / \mathrm{ml} \mathrm{PI}$ and $20 \mu \mathrm{g} / \mathrm{ml}$ DAPI were added directly to the media. After this incubation, the MIN6 cells were washed 3 times with PBS and fixed with 3.7\% formaldehyde for 15 minutes at $4{ }^{\circ} \mathrm{C}$. Each condition reported represents greater than 600 cells counted by randomized field selection. The percentage of cell death was calculated as the number of PI-stained nuclei per the total number of nuclei stained by DAPI.
MIN6 cell and islet culture and Pdx1 suppression. Methods for culture of MIN6 cells (35) and islets and the recombinant lentivirus system used to deliver shRNAs to MIN6 cells were previously reported $(15,16)$.

Immunoblot analysis. Blots were probed with antibodies against $\mathrm{Pdx} 1$ (catalog no. sc-14664; Santa Cruz Biotechnology Inc.), cleaved caspase-3 (catalog no. 9661; Cell Signaling Technologies), actin (catalog no. A-2066; Sigma-Aldrich), Nix (catalog no. ab8399; Abcam Inc.), LC3 (catalog no. NB100-2331; Novus Biologicals Inc.), Flag (catalog no. F-3165; SigmaAldrich), cytochrome c (catalog no. AP1029; Calbiochem), and COX IV (catalog no. ab14744; Abcam Inc.).

Mitochondrial isolation. MIN6 cells were homogenized and subjected to centrifugation at $3,000 \mathrm{~g}$ for 10 minutes to remove nuclei and myofibrils. The supernatant was then subjected to centrifugation at $10,000 \mathrm{~g}$ for 10 minutes. The resultant pellet and supernatant were labeled as $10 \mathrm{p}$ (mitochondrial enriched) fraction and 10s (cytosol) fractions.

Quantification of $m R N A$ level. RNA isolation, first strand cDNA synthesis, and TaqMan gene expression assays were performed as previously described $(15,16)$. Applied Biosystems TaqMan assay numbers were as follows: $\mathrm{Hmbs}$, Mm00660262_g1; Pdx1, Mm00435565_m1; Nix, Mm00786306_s1.

Measurement of $\Delta \psi_{m}$. Rhodamine 123 was used as an indicator of $\Delta \psi_{\mathrm{m}}$. This fluorescent lipophilic cationic dye is in the mitochondrial matrix because of its charge and solubility in both the inner mitochondrial membrane and matrix space (36). In the present study, MIN6 cells or dispersed islets were loaded by incubation with $10 \mu \mathrm{g} / \mathrm{ml}$ rhodamine 123 for 20 minutes at $37^{\circ} \mathrm{C}$. Rhodamine 123 fluorescence was excited at $490 \mathrm{~nm}$ and was measured at $530 \mathrm{~nm}$. Mean fluorescence and cell counts were assayed by FACS analysis.

Lentivirus-mediated shRNA expression and Ad infection studies. shRNA targeting mouse Nix was obtained from Sigma-Aldrich. Recombinant lentiviral particles were prepared as previously described $(15,16)$. Recombinant Ads were created by cloning Flag-tagged Nix into pAdEasy-1 vector (Stratagene) using pShuttleCMV and by recombination in BJ5183-AD-1 cells as previously described (22). MIN6 cells and isolated mouse islets were infected with Ads at a titer of $10 \mathrm{PFU} /$ cell. Efficiency of Ad infection to the islets was determined on isolated islets from WT mice that were incubated with Ad-containing media for 2 days. Efficiency of Ad infection was examined on cryosections $(10 \mu \mathrm{m})$ of fixed islets with an anti-Flag antibody (catalog no. F-3165; Sigma-Aldrich).

In vivo characterization of mice. The $P d x 1^{+/-}$mice have been previously described (5) and were provided by H. Edlund (University of Umea, Umea, Sweden). $\mathrm{Nix}^{-1}$ mice have been described previously (21). Male mice were fed a high-fat diet containing $42 \%$ fat (Harlan Laboratories Inc.) from 3 weeks of age and provided with water ad libitum. Intraperitoneal glucose tolerance tests were performed after a 4-hour fast ( $1 \mathrm{~g} / \mathrm{kg}$ dextrose). Insulin levels were measured at fasting and 30 minutes after glucose challenge. Insulin tolerance tests were performed after a 4-hour fast by administering human recombinant insulin $(0.75 \mathrm{U} / \mathrm{kg})$. We quantified $\beta$ cell area from anti-insulin-stained pancreas sections counterstained with hematoxylin using the intensity thresholding function of the integrated morphometry package in MetaMorph. TUNEL labeling used the DeadEnd Fluorometric TUNEL System (Promega Corp.). All experiments in this study using animal protocols were approved by the Washington University Animal Studies Committee.

Confocal imaging studies of pancreatic islets. Formalin-fixed pancreas sections underwent antigen retrieval in boiling citrate buffer ( $\mathrm{pH}$ 6.0) for 10 minutes before labeling with antibodies against insulin (catalog no. 4010-01L; Millipore) and glucagon (catalog no. G2654; Sigma-Aldrich). Images were obtained on a Nikon Personal Confocal microscope.

Statistics. Partek software was used to compute significance of mRNA expression changes in microarray using 1 -way ANOVA at $P<0.01$. Multiple experimental groups were compared using 1-way ANOVA and Tukey's posthoc test. The 2-tailed unpaired Student's $t$ test was used to assess the statistical 
significance of differences between 2 sets of data. Differences were considered significant when $P<0.05$. Results are presented as mean \pm SEM.

\section{Acknowledgments}

This work was supported by NIH grants R01 HL059888 (to G.W. Dorn) and R01 DK031842 (to K.S. Polonsky), the Radioimmunoassay and Morphology Cores of the Diabetes Research and Training Center of Washington University (P60 DK-20579), the Washington University Digestive Diseases Research Center Morphology Core (P30 DK052574), the Clinical and Translational Science Award to Washington University (UL1 RR024992), and the Blum Kovler Foundation.
Received for publication June 14, 2010, and accepted in revised form September 1, 2010.

Address correspondence to: Kenneth S. Polonsky, Department of Medicine, Washington University School of Medicine, Campus Box 8066, 660 S. Euclid Avenue, St. Louis, Missouri 63110, USA. Phone: 314.362.8061; Fax: 314.362.8015; E-mail: Polonsky@ dom.wustl.edu. Or to: Gerald W. Dorn II, Washington University Center for Pharmacogenomics, Campus Box 8220, 660 S. Euclid Ave., St. Louis, Missouri 63110, USA. Phone: 314.362.4892; Fax: 314.362.8844; E-mail: gdorn@dom.wustl.edu.
1. Stoffers DA, Zinkin NT, Stanojevic V, Clarke WL, Habener JF. Pancreatic agenesis attributable to a single nucleotide deletion in the human IPF1 gene coding sequence. Nat Genet. 1997;15(1):106-110.

2. Stoffers DA, Stanojevic V, Habener JF. Insulin promoter factor-1 gene mutation linked to earlyonset type 2 diabetes mellitus directs expression of a dominant negative isoprotein. J Clin Invest. 1998; 102(1):232-241.

3. Macfarlane WM, et al. Missense mutations in the insulin promoter factor-1 gene predispose to type 2 diabetes. J Clin Invest. 1999;104(9):R33-R39.

4. Elbein SC, Das SK, Hallman DM, Hanis CL, Hasstedt SJ. Genome-wide linkage and admixture mapping of type 2 diabetes in African American families from the American Diabetes Association GENNID (Genetics of NIDDM) Study Cohort. Diabetes. 2009;58(1):268-274

5. Jonsson J, Carlsson L, Edlund T, Edlund H. Insulinpromoter-factor 1 is required for pancreas development in mice. Nature. 1994;371(6498):606-609.

6. Ahlgren U, Jonsson J, Edlund H. The morphogenesis of the pancreatic mesenchyme is uncoupled from that of the pancreatic epithelium in IPF1/PDX1-deficient mice. Development. 1996; 122(5):1409-1416.

7. Offield MF, et al. PDX-1 is required for pancreatic outgrowth and differentiation of the rostral duodenum. Development. 1996;122(3):983-995.

8. Sharma A, et al. The homeodomain protein IDX-1 increases after an early burst of proliferation during pancreatic regeneration. Diabetes. 1999; 48(3):507-513.

9. Hart AW, Baeza N, Apelqvist A, Edlund H. Attenuation of FGF signaling in mouse beta-cells leads to diabetes. Nature. 2000;408(6814):864-868.

10. Gannon $M$, et al. pdx-1 function is specifically required in embryonic beta cells to generate appropriate numbers of endocrine cell types and maintain glucose homeostasis. Dev Biol. 2008;314(2):406-417.

11. Brissova M, et al. Reduction in pancreatic transcription factor PDX-1 impairs glucose-stimulated insulin secretion. J Biol Chem. 2002;277(13):11225-11232.

12. Johnson JD, et al. Increased islet apoptosis in $P d x 1^{+/-}$mice. J Clin Invest. 2003;111(8):1147-1160.

13. Johnson JD, et al. Insulin protects islets from apoptosis via $\mathrm{Pdx} 1$ and specific changes in the human islet proteome. Proc Natl Acad Sci U S A. 2006; 103(51):19575-19580.

14. Li Y, Cao X, Li LX, Brubaker PL, Edlund H, Drucker DJ. beta-Cell Pdx1 expression is essential for the glucoregulatory, proliferative, and cytoprotective actions of glucagon-like peptide- 1 . Diabetes. 2005;54(2):482-491.

15. Fujimoto K, et al. Autophagy regulates pancreatic beta cell death in response to $\mathrm{Pdx} 1$ deficiency and nutrient deprivation. J Biol Chem. 2009; 284(40):27664-27673.

16. Fujimoto K, Chen Y, Polonsky KS, Dorn GW 2nd. Targeting cyclophilin D and the mitochondrial permeability transition enhances beta-cell survival and prevents diabetes in $\mathrm{Pdx} 1$ deficiency. Proc Natl Acad Sci U S A. 2010;107(22):10214-10219.

17. Butler PC, Meier JJ, Butler AE, Bhushan A. The replication of beta cells in normal physiology, in disease and for therapy. Nat Clin Pract Endocrinol Metab. 2007;3(11):758-768.

18. Chen G, et al. Nix and Nip3 form a subfamily of pro-apoptotic mitochondrial proteins. J Biol Chem. 1999;274(1):7-10.

19. Ray R, et al. BNIP3 heterodimerizes with Bcl-2/ $\mathrm{Bcl}-\mathrm{X}(\mathrm{L})$ and induces cell death independent of a Bcl-2 homology 3 (BH3) domain at both mitochondrial and nonmitochondrial sites.J Biol Chem. 2000;275(2):1439-1448.

20. Sandoval $\mathrm{H}$, et al. Essential role for Nix in autophagic maturation of erythroid cells. Nature. 2008;454(7201):232-235.

21. Diwan A, et al. Unrestrained erythroblast development in $\mathrm{Nix}^{-/-}$mice reveals a mechanism for apoptotic modulation of erythropoiesis. Proc Natl Acad Sci US A. 2007;104(16):6794-6799.

22. Diwan A, et al. Endoplasmic reticulum-mitochondria crosstalk in NIX-mediated murine cell death. J Clin Invest. 2009;119(1):203-212.

23. Chen Y, Lewis W, Diwan A, Cheng EH, Matkovich SJ, Dorn GW 2nd. Dual autonomous mitochondrial cell death pathways are activated by Nix/BNip3L and induce cardiomyopathy. Proc Natl Acad SciUS A. 2010;107(20):9035-9042.

24. Dorn GW 2nd. Having a change of heart: reversing the suicidal proclivities of cardiac myocytes. Trans Am Clin Climatol Assoc. 2009;120:189-198.

25. Zhang J, Ney PA. Role of BNIP3 and NIX in cell death, autophagy, and mitophagy. Cell Death Differ. 2009;16(7):939-946.

26. Kulkarni RN, Jhala US, Winnay JN, Krajewski S, Montminy M, Kahn CR. PDX-1 haploinsufficiency limits the compensatory islet hyperplasia that occurs in response to insulin resistance. J Clin Invest. 2004;114(6):828-836.

27. Schweers RL, et al. NIX is required for programmed mitochondrial clearance during reticulocyte maturation. Proc Natl Acad Sci U S A. 2007; 104(49):19500-19505.

28. Keller DM, et al. Characterization of pancreatic transcription factor $\mathrm{Pdx}-1$ binding sites using promoter microarray and serial analysis of chromatin occupancy. J Biol Chem. 2007;282(44):32084-32092.

29. Gunton JE, et al. Loss of ARNT/HIF1beta mediates altered gene expression and pancreatic-islet dysfunction in human type 2 diabetes. Cell. 2005; 122(3):337-349.

30. Gálvez AS, et al. Distinct pathways regulate proapoptotic Nix and BNip3 in cardiac stress. J Biol Chem. 2006;281(3):1442-1448.

31. Bruick RK. Expression of the gene encoding the proapoptotic Nip3 protein is induced by hypoxia. Proc Natl Acad Sci U S A. 2000;97(16):9082-9087.

32. Sowter HM, Ratcliffe PJ, Watson P, Greenberg AH, Harris AL. HIF-1-dependent regulation of hypoxic induction of the cell death factors BNIP3 and NIX in human tumors. Cancer Res. 2001;61(18):6669-6673.

33. Bakker WJ, Harris IS, Mak TW. FOXO3a is activated in response to hypoxic stress and inhibits HIF1induced apoptosis via regulation of CITED2. Mol Cell. 2007;28(6):941-953.

34. Matkovich SJ, et al. MicroRNA-133a protects against myocardial fibrosis and modulates electrical repolarization without affecting hypertrophy in pressure-overloaded adult hearts. Circ Res. 2010;106(1):166-175.

35. Miyazaki J, et al. Establishment of a pancreatic beta cell line that retains glucose-inducible insulin secretion: special reference to expression of glucose transporter isoforms. Endocrinology. 1990; 127(1):126-132.

36. Scaduto RC Jr, Grotyohann LW. Measurement of mitochondrial membrane potential using fluorescent rhodamine derivatives. Biophys J. 1999; $76(1$ pt 1$): 469-477$ 